\title{
Ascertaining Weather Indices to Exploit the Yield Potential of Chickpea (Cicer arietinum L.) at Scarce Rainfall Zone of Andhra Pradesh
}

\author{
K. Prabhakar ${ }^{1 *}$, V. Sumathi ${ }^{1}$, T. Giridhar Krishna ${ }^{2}$, P. Sudhakar ${ }^{3}$, \\ S. Jaffar Basha ${ }^{4}$ and G. Karuna Sagar ${ }^{1}$ \\ ${ }^{1}$ Department of Agronomy, S. V. Agricultural College, Tirupati, ANGRAU, A.P., India \\ ${ }^{2}$ ANGRAU, Lam, Guntur, A.P., India \\ ${ }^{3}$ Crop physiology, RARS, Tirupathi, A.P., India \\ ${ }^{4}$ Agronomy, AINP on Tobacco, RARS Nandyal, Kurnool District, A.P., India \\ *Corresponding author
}

\begin{tabular}{l} 
K e y w o r d s \\
Chickpea, \\
Phenophases, GDD, \\
PTU, HTU and \\
TUE \\
\hline Article Info \\
$\begin{array}{l}\text { Accepted: } \\
12 \text { October } 2020 \\
\text { Available Online: } \\
10 \text { November } 2020\end{array}$ \\
\hline
\end{tabular}

\section{A B S T R A C T}

Field experiments were conducted during rabi season of 2018-19 and 2019-20 at ANGRAU, Regional Agricultural Research Station, Nandyal, to study the weather related information on chickpea (Cicer arietinum L.) growth and yield under crop residue incorporation, varied time of sowing and irrigation stages. Results revealed that the mean values of weather indices like accumulated growing degree day, helio thermal units, photo thermal units at different phenophases and thermal use efficiency were influenced by times of sowing but not with crop residues incorporation and irrigation. Time of sowing also did not influence the days to reach different phenophases from emergence to harvest significantly. However, the first and second dates of sowing recorded more number of days to reach 50 per cent flowering, physiological maturity and harvest stage. Growing degree days, helio thermal units, photo thermal units, at flowering, physiological maturity and harvesting maturity of crop found to be highest in October second fortnight sown crop and goes on decreased with delay in sowing with fortnight interval, up to December first fort night. The drymatter and seed yield were higher with November first fortnight sown crop drymatter production $\left(4719 \mathrm{~kg} \mathrm{ha}^{-1}\right)$, seed yield $\left(1660 \mathrm{~kg} \mathrm{ha}^{-1}\right)$. The study concludes that maximum seed yield of chickpea can be achieved when chickpea was sown during November $1^{\text {st }}$ FN, with incorporation of foxtail millet crop residue with two irrigations, one at pre flowering and another at pod development stage, attributed to record the higher thermal use efficiency (TUE) (dry matter $2.25 \mathrm{~kg}$ ha ${ }^{-1}{ }^{0} \mathrm{C}$ day and seed $0.77 \mathrm{~kg}$ ha ${ }^{-1}{ }^{0} \mathrm{C}$ day) under SRZ of Andhra Pradesh.

\section{Introduction}

Chickpea is a drought tolerant, photoperiod sensitive $\mathrm{C}_{3}$ plant originated in Turkish
Kurdistan (Lev et al., 2000). In sub-tropical region like India, the climate is temperate with kharif rainfall mostly from JuneSeptember. Chickpea is conventionally grown 
during winter, based on available residual soil moisture on deep clayey soils. Therefore, the crop faces high temperature and water stress towards maturity which result in low and variable yields.

Chickpea is a thermo-sensitive winter season crop. The most important factors affecting chickpea are temperature and photoperiod (Summerfield et al., 1980; Sandhu and Hodges, 1971; Kiran and Chimmad, 2015). In the view of recent climate change situation, the weather parameters are highly influencing the crop productivity wherein, there is an increase in day temperature and drastic reduction in the night temperature and photo periods are expecting in future days.

Time of sowing is an important agronomic factor affecting the productivity of most of the arable crops, owing to changes in environmental conditions to which phenological stages of crops are exposed. Under late sown conditions, the growth of chickpea is affected resulting in low yield. Variable seed yields are a deterrent to growing chickpea (Cicer arietinum L.) as they are mostly grown on residual soil moisture and often experience water stress during their terminal growth in major chickpea growing countries.

Determining the moisture basis for yield variation may help to determine best management practices to maximize yield, which may help to identify other areas as potential production sites. Dixith et al., (1993) reported that earlier or late sowing of chickpea caused drastic reduction in yield and net profit compared with timely sowing. Mohammed et al., (2017) concluded that when sowing time for chickpea is delayed the residual soil moisture available to support the crop growth might be depleted onwards, which could expose the crop for terminal drought and finally reduce the grain yield, on other hand, sowing too early may expose the crop to water logging which also a major problem for chickpea production particularly in vertisols. So it is appropriate to find optimum time of sowing for different farming situations under double cropping system to improve yields and net returns.

\section{Materials and Methods}

Field experiments were carried out for two consecutive kharif and rabi seasons of 201819 and 2019-20 at R.A.R.S. Farm, Nandyal, Andhra Pradesh. The treatments comprised of three crop residue incorporations viz., foxtail millet $\left(\mathrm{C}_{1}\right)$, greengram $\left(\mathrm{C}_{2}\right)$ and fallow $\left(\mathrm{C}_{3}\right)$ as main plot treatments and four times of sowing viz. October $2^{\text {nd }} \mathrm{FN}\left(\mathrm{D}_{1}\right)$, November $1^{\text {st }} \mathrm{FN}$ $\left(\mathrm{D}_{2}\right)$, November $2^{\text {nd }} \mathrm{FN}\left(\mathrm{D}_{3}\right)$ and December $1^{\text {st }} \mathrm{FN}\left(\mathrm{D}_{4}\right)$ as sub plot treatments and three irrigation schedules as sub- sub plots with irrigation at pre-flowering stage $\left(\mathrm{I}_{1}\right)$, irrigation at pod development stage $\left(\mathrm{I}_{2}\right)$ and irrigation at pre-flowering and pod development stage $\left(\mathrm{I}_{3}\right)$ During kharif season, foxtail millet and greengram crops were raised as bulk crops in respective main plots and crop residues were incorporated after harvest of economic parts viz., panicles of foxtail millet and pods of greengram. Experimental design was splitsplit plot, with three replications.

The site was situated at an altitude of $216 \mathrm{~m}$ above mean sea level at $15^{\circ} 29^{\prime} 19^{\prime}$ ' $\mathrm{N}$ latitude and $78^{\circ} 29^{\prime} 11^{\prime}$ ' E longitude, mostly under rainfed conditions, categorized in the Scarce rainfall Agro-climatic Zone of Andhra Pradesh. The meteorological data of maximum and minimum temperature, rainfall, rainy days, morning and evening relative humidity and wind speed were recorded from meteorological observatory, Regional Agricultural Research station, Nandyal near the experimental site, during the period of crop growth. Soil of the site was medium in fertility and slightly saline in reaction having 
$\mathrm{pH} 8.42$, electrical conductivity $0.24 \mathrm{dSm}^{-1}$, organic carbon $0.32 \%$ with available nitrogen, phosphorus and potassium of N, 143, 53 and $451 \mathrm{~kg} / \mathrm{ha}$, respectively. Sowing of seeds was done in rows, $30 \mathrm{~cm}$ apart with $10 \mathrm{~cm}$ between plants. An amount of $20 \mathrm{~kg}$ nitrogen and $50 \mathrm{~kg} \mathrm{P}_{2} \mathrm{O}_{5}$ per hectare was applied through urea and single SSP in basal. Sowing was done in four intervals as $\mathrm{D}_{1}$ on October $2^{\text {nd }}$ fortnight, $D_{2}$ on November $1^{\text {st }}$ fortnight, $\mathrm{D}_{3}$ on November $2^{\text {nd }}$ fortnight, $\mathrm{D}_{4}$ on December $1^{\text {st }}$ fortnight, in respective treatment plots. Healthy and matured seeds NBeG-3 chickpea desi variety having high germination percentage was used for sowing. Seed rate@ @ $50 \mathrm{~kg} \mathrm{ha}^{-1}$ was adopted and sown in the open furrows made with the help of hand hoe. The seeds were dropped to a depth of $5 \mathrm{~cm}$ and covered thoroughly. The phonological development of the crop was monitored at 2- 3 days interval to decide the duration taken to reach different physiological stages, where $50 \%$ of plumule emergence was considered as days to emergence, $50 \%$ plants with one flower at any node was considered as days flowering, $95 \%$ of pods had obtained their mature colour was considered as physiological maturity and harvest stage was when all ground parts attains matured straw colour (Soltani et al., 2006). Agrometeorological indices like Growing Degree Days (GDD), Heliothermal Units (HTU), Photothermal Units (PTU) and Thermal use efficiency (TUE) were computed by adopting the procedure laid out by Rajput (1980).

\section{Growing degree days}

The growing degree-days (GDD) were determined as (Nuttonson, 1955)

\section{GDD $=$}

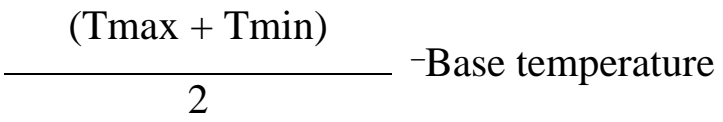

Where, Tmax and Tmin are the daily maximum and minimum temperature $\left({ }^{0} \mathrm{C}\right)$. Base temperature of $5{ }^{\mathrm{O}} \mathrm{C}$ was adopted for calculation.

\section{Helio thermal units}

The helio thermal units for a given day represent the product of GDD and the actual hours of bright sun shine for that day. The sum of the HTU for the duration of each phenophase was determined by using the formula.

Accumulated HTU $\left({ }^{\circ} \mathrm{C}\right.$ day hour $)=$ GDD $\times$ Duration of sunshine hours

\section{Photo thermal units}

The photo thermal units for each day represent the product of GDD and the day length. The accumulated PTU for each phenophase was determined by the following formula.

Accumulated PTU $\left({ }^{\circ} \mathrm{C}\right.$ day hour $)=$ GDD $\times$ Day length

\section{Thermal Use Efficiency (TUE)}

The TUE was worked using the formula and a unit for heat use efficiency is $\mathrm{kg} \mathrm{ha}^{-1}$ per degree day.

Thermal use efficiency $($ TUE $)=$

\section{Seed yield/dry matter}

Growing degree days

Five randomly selected plants outside the net plot area collected at 30, 60 DAS and at harvest were used for estimation of dry matter production. These samples were air dried and then oven dried at $60^{\circ} \mathrm{C}$ to a constant weight and expressed in $\mathrm{kg} \mathrm{ha}^{-1}$. The seed yield obtained from net plot area was threshed, 
cleaned and sundried and expressed as $\mathrm{kg} \mathrm{ha}^{-1}$.

\section{Results and Discussion}

Results pertaining to effect of crop residue incorporation, time of sowing and irrigation stages on days to reach different phenophases, accumulated GDD, HTU, PTU, TUE and seed yield were presented in table number 1 to 5 and Fig. 1. Results shown yearly variations in all weather indices studied.

\section{Days to reach different phenophases}

Response of chickpea to crop residue incorporation, time of sowing and irrigation to reach different phenophases (days) during rabi 2018-19 and 2019-20 is presented in Table 1. The results indicated that crop residue incorporation did not influence significantly the days to reach different phenophases from emergence to harvest. However, during both the years of study seedling emergence was slightly delayed in crop residue incorporated plots compared to fallow. Thereafter, 50 per cent flowering, physiological maturity and harvest was relatively faster in crop residue incorporated plots then in fallow plots.

Time of sowing also did not influence the days to reach different phenophases from emergence to harvest significantly. However, the first and second dates of sowing recorded more number of days to reach 50 per cent flowering, physiological maturity and harvest stage. It was observed that during both the years, gradual delay in time of sowing from October month to December month decreased the days to reach different phenophases. This might be due to exposing of late sown crop with increased temperatures in their later growth stages.

The interaction between crop residue incorporation, time of sowing and irrigation levels either at two level or three level interaction was not significant in altering the duration of phenophases, during the both the years of study.

In the present investigation, delay in sowing from October second fortnight to December first fort night, reduced the number of days to attain physiological maturity and harvesting maturity of crop. These results are in accordance with the results reported by Agarwal and Upadyaya (2016). It is mainly due to increasing temperature at reproductive phase those of which had profound effect on phenology of chickpea.

\section{Growing degree days (GDD)}

The accumulated growing degree days attained by chickpea at different phenophases under different treatments were calculated and presented in Table 2.

The GDD were influenced by time of sowing only but not with crop residues and irrigation during both the years of study. Accumulated GDD values from sowing to emergence were higher in October $2^{\text {nd }}$ FN sowing i.e same $140^{\circ} \mathrm{C}$ day, during 2018-19 and 2019-20 respectively followed by November $1^{\text {st }}$ FN sowing and gradually reduced with delay in sowing upto December $1^{\text {st }}$ FN sowing i.e. 81.5 and $80.6{ }^{\circ} \mathrm{C}$ day, during $2018-19$ and 2019-20, respectively.

Growing degree days (GDD) value at flowering, physiological maturity and harvesting maturity of crop found to be highest in $1^{\text {st }}$ growing environments and goes on decreasing with $2^{\text {nd }} 3^{\text {rd }}$ and fourth growing environments, due to shorter phonological stages with delay in sowing. Sunil Kumar and Martin Luther, (2018) and Sada Kumar et al., (2018) were also reported similar line of results. 
Table.1 Days to reach different phenophases of chickpea as influenced by crop residue incorporation, time of sowing and Irrigation

\begin{tabular}{|c|c|c|c|c|c|c|c|c|}
\hline \multirow[t]{2}{*}{ Treatment } & \multicolumn{2}{|c|}{ Emergence * } & \multicolumn{2}{|c|}{$\begin{array}{c}\text { Fifty \% } \\
\text { flowering }\end{array}$} & \multicolumn{2}{|c|}{$\begin{array}{c}\text { Physiological } \\
\text { maturity }\end{array}$} & \multicolumn{2}{|c|}{ Harvest } \\
\hline & $\begin{array}{l}\text { Rabi, } \\
2018\end{array}$ & $\begin{array}{l}\text { Rabi, } \\
2019\end{array}$ & $\begin{array}{l}\text { Rabi, } \\
2018\end{array}$ & $\begin{array}{l}\text { Rabi, } \\
2019\end{array}$ & $\begin{array}{l}\text { Rabi, } \\
2018\end{array}$ & $\begin{array}{l}\text { Rabi, } \\
2019\end{array}$ & $\begin{array}{l}\text { Rabi, } \\
2018\end{array}$ & $\begin{array}{l}\text { Rabi, } \\
2019\end{array}$ \\
\hline \multicolumn{9}{|l|}{ Crop residue incorporation } \\
\hline $\mathrm{C}_{1}$ : Foxtail millet & 5.2 & 5.3 & 39.1 & 41.3 & 94 & 96 & 101 & 104. \\
\hline $\mathrm{C}_{2}$ : Greengram & 4.7 & 5.2 & 39.0 & 41.1 & 95 & 96 & 100 & 105 \\
\hline $\mathrm{C}_{3}:$ Fallow & 4.3 & 4.7 & 38.3 & 40.3 & 94 & 96 & 100 & 103 \\
\hline SEm \pm & - & - & 1.11 & 0.35 & 0.7 & 0.9 & 0.7 & 0.9 \\
\hline $\mathrm{CD}(\mathrm{P}=\mathbf{0 . 0 5})$ & - & -- & NS & NS & NS & NS & NS & NS \\
\hline \multicolumn{9}{|l|}{ Time of sowing } \\
\hline$D_{1}:$ October $2^{\text {nd }}$ FN & 5.3 & 5.5 & 40.7 & 42.2 & 97 & 98 & 104 & 107 \\
\hline $\mathrm{D}_{2}:$ November $1^{\text {st }} \mathrm{FN}$ & 4.9 & 4.8 & 40.5 & 41.9 & 95 & 97 & 104 & 105 \\
\hline$D_{3}$ : November $2^{\text {nd }}$ FN & 4.7 & 4.6 & 37.4 & 41.2 & 95 & 98 & 99 & 101 \\
\hline $\mathrm{D}_{4:}$ December $1^{\text {st }} \mathrm{FN}$ & 4.7 & 4.6 & 36.3 & 40.1 & 90 & 95 & 94 & 97 \\
\hline SEm \pm & - & - & 2.62 & 0.96 & 1.2 & 1.2 & 3.6 & 3.3 \\
\hline $\mathrm{CD}(\mathrm{P}=\mathbf{0 . 0 5})$ & - & - & NS & NS & NS & NS & NS & NS \\
\hline \multicolumn{9}{|l|}{ Time of irrigation } \\
\hline$I_{1}$ : Irrigation at pre-flowering stage & - & - & 38.6 & 39.5 & 95 & 95 & 100 & 102 \\
\hline$I_{2}:$ Irrigation at pod development stage & - & - & 38.6 & 39.4 & 94 & 96 & 101 & 102 \\
\hline $\begin{array}{l}\text { I3: Irrigation at pre-flowering and pod } \\
\text { development stage }\end{array}$ & & - & 39.6 & 39.2 & 95 & 95 & 101 & 103 \\
\hline $\mathrm{SEm} \pm$ & - & - & 1.25 & 0.52 & 0.7 & 0.5 & 1.1 & 1.2 \\
\hline $\mathrm{CD}(\mathrm{P}=\mathbf{0 . 0 5})$ & - & - & NS & NS & NS & NS & NS & NS \\
\hline
\end{tabular}

\footnotetext{
*Statistically not analysed
} 
Table.2 Accumulated Growing degree days $\left({ }^{0} \mathrm{C}\right.$ day $)$ at different phenophases of chickpea as influenced by crop residue incorporation, time of sowing and irrigation

\begin{tabular}{|c|c|c|c|c|c|c|c|c|c|}
\hline \multirow[t]{2}{*}{ Treatment } & \multicolumn{2}{|c|}{ Emergence * } & \multicolumn{2}{|c|}{$\begin{array}{l}\text { Fifty \% } \\
\text { flowering }\end{array}$} & \multicolumn{2}{|c|}{$\begin{array}{l}\text { Physiological } \\
\text { maturity }\end{array}$} & \multicolumn{3}{|c|}{ Harvest } \\
\hline & $\begin{array}{r}\text { Rabi, } \\
2018\end{array}$ & $\begin{array}{l}\text { Rabi, } \\
2019\end{array}$ & $\begin{array}{r}\text { Rabi, } \\
2018\end{array}$ & $\begin{array}{l}\text { Rabi, } \\
2019\end{array}$ & $\begin{array}{l}\text { Rabi, } \\
2018\end{array}$ & $\begin{array}{l}\text { Rabi, } \\
2019\end{array}$ & $\begin{array}{l}\text { Rabi, } \\
2018\end{array}$ & $\begin{array}{l}\text { Rabi, } \\
2019\end{array}$ & Pooled \\
\hline \multicolumn{10}{|l|}{ Crop residue incorporation } \\
\hline $\mathrm{C}_{1}:$ Foxtail millet & 101 & 101 & 820 & 825 & 1952 & 1955 & 2073 & 2075 & 2074 \\
\hline $\mathrm{C}_{2}$ : Greengram & 101 & 102 & 820 & 826 & 1953 & 1954 & 2073 & 2076 & 2074 \\
\hline $\mathrm{C}_{3}$ : Fallow & 101 & 101 & 818 & 821 & 1951 & 1954 & 2071 & 2074 & 2072 \\
\hline $\mathrm{SEm} \pm$ & - & - & 1.3 & 0.9 & 2.3 & 1.0 & 2.2 & 0.9 & 0.9 \\
\hline $\mathrm{CD}(\mathrm{P}=\mathbf{0 . 0 5})$ & - & - & NS & NS & NS & NS & NS & NS & NS \\
\hline \multicolumn{10}{|l|}{ Time of sowing } \\
\hline $\mathrm{D}_{1}:$ October $2^{\text {nd }} \mathrm{FN}$ & 140 & 140 & 910 & 924 & 2072 & 2092 & 2172 & 2192 & 2182 \\
\hline$D_{2}:$ November $1^{\text {st }} F N$ & 92 & 92 & 877 & 855 & 2017 & 1992 & 2117 & 2092 & 2105 \\
\hline$D_{3}:$ November $2^{\text {nd }} F N$ & 91 & 88 & 807 & 839 & 1929 & 1974 & 2029 & 2074 & 2051 \\
\hline $\mathrm{D}_{4:}$ December $1^{\text {st }} \mathrm{FN}$ & 82 & 81 & 720 & 706 & 1827 & 1818 & 1927 & 1918 & 1922 \\
\hline SEm \pm & - & - & 12.6 & 11.5 & 16.3 & 10.4 & 16.4 & 10.3 & 11.5 \\
\hline $\mathrm{CD}(\mathrm{P}=\mathbf{0 . 0 5})$ & - & - & 37 & 34 & 46 & 31 & 46 & 31 & 35 \\
\hline \multicolumn{10}{|l|}{ Time of irrigation } \\
\hline$I_{1}:$ Irrigation at pre-flowering stage & - & - & 818 & 821 & 1951 & 1952 & 1961 & 1962 & 1962 \\
\hline$I_{2}:$ Irrigation at pod development stage & - & - & 819 & 823 & 1951 & 1953 & 1961 & 1963 & 1962 \\
\hline $\begin{array}{l}\text { I3: Irrigation at pre-flowering and pod } \\
\text { development stage }\end{array}$ & - & - & 828 & 824 & 1960 & 1956 & 1969 & 1966 & 1968 \\
\hline SEm \pm & - & - & 3.3 & 1.0 & 14.2 & 2.4 & 14.3 & 2.3 & 3.4 \\
\hline $\mathrm{CD}(\mathrm{P}=\mathbf{0 . 0 5})$ & - & - & NS & NS & NS & NS & NS & NS & NS \\
\hline
\end{tabular}

*Statistically not analysed 
Table.3 Accumulated Helio thermal units $\left({ }^{0} \mathrm{C}\right.$ day hrs $)$ at different phenophases of chickpea as influenced by crop residue incorporation, time of sowing and irrigation

\begin{tabular}{|c|c|c|c|c|c|c|c|c|c|}
\hline \multirow[t]{2}{*}{ Treatment } & \multicolumn{2}{|c|}{ Emergence* } & \multicolumn{2}{|c|}{ Fifty \% flowering } & \multicolumn{2}{|c|}{$\begin{array}{l}\text { Physiological } \\
\text { maturity }\end{array}$} & \multicolumn{3}{|c|}{ Harvest } \\
\hline & $\begin{array}{l}\text { Rabi, } \\
2018\end{array}$ & $\begin{array}{l}\text { Rabi, } \\
2019\end{array}$ & $\begin{array}{r}\text { Rabi, } \\
2018\end{array}$ & $\begin{array}{r}\text { Rabi, } \\
2019\end{array}$ & $\begin{array}{r}\text { Rabi, } \\
2018\end{array}$ & $\begin{array}{r}\text { Rabi, } \\
2019\end{array}$ & $\begin{array}{r}\text { Rabi, } \\
2018\end{array}$ & $\begin{array}{l}\text { Rabi, } \\
2019\end{array}$ & Pooled \\
\hline \multicolumn{10}{|l|}{ Crop residue incorporation } \\
\hline $\mathrm{C}_{1}$ : Foxtail millet & 748 & 628 & 6031 & 5607 & 15325 & 14586 & 16125 & 16185 & 16155 \\
\hline $\mathrm{C}_{2}$ : Greengram & 751 & 629 & 6037 & 5606 & 15322 & 14586 & 16122 & 1618 & 16154 \\
\hline $\mathrm{C}_{3}$ : Fallow & 754 & 626 & 6041 & 5602 & 15321 & 14580 & 16120 & 16181 & 16149 \\
\hline $\mathrm{SEm} \pm$ & - & - & 2.4 & 2.3 & 2.3 & 2.1 & 2.3 & 2.1 & 3.6 \\
\hline $\mathrm{CD}(\mathrm{P}=\mathbf{0 . 0 5})$ & - & - & NS & NS & NS & NS & NS & NS & NS \\
\hline \multicolumn{10}{|l|}{ Time of sowing } \\
\hline $\mathrm{D}_{1}:$ October $2^{\text {nd }} \mathrm{FN}$ & 1331 & 899 & 7726 & 6817 & 19548 & 17801 & 20399 & 18652 & 19526 \\
\hline$D_{2}:$ November $1^{\text {st }} F N$ & 819 & 572 & 6704 & 6445 & 16637 & 16046 & 17488 & 16897 & 17191 \\
\hline$D_{3}:$ November $2^{\text {nd }} F N$ & 625 & 625 & 5150 & 5588 & 13777 & 14469 & 14628 & 15320 & 14974 \\
\hline $\mathrm{D}_{4:}$ December $1^{\text {st }} \mathrm{FN}$ & 423 & 348 & 4760 & 5101 & 13213 & 12689 & 14063 & 13540 & 13801 \\
\hline $\mathrm{SEm} \pm$ & - & - & 7.8 & 6.3 & 11.6 & 9.3 & 11.6 & 9.3 & 11.2 \\
\hline $\mathrm{CD}(\mathrm{P}=0.05)$ & - & - & 23 & 19 & 35 & 28 & 35 & 28 & 33 \\
\hline \multicolumn{10}{|l|}{ Time of irrigation } \\
\hline$I_{1}$ : Irrigation at pre-flowering stage & - & - & 6026 & 5609 & 15308 & 14575 & 16108 & 15375 & 15741 \\
\hline $\begin{array}{l}\mathrm{I}_{2}: \text { Irrigation at pod development } \\
\text { stage }\end{array}$ & - & - & 6020 & 5614 & 15307 & 14583 & 16108 & 15385 & 15745 \\
\hline $\begin{array}{l}\text { I3: Irrigation at pre-flowering and pod } \\
\text { development stage }\end{array}$ & - & - & 6062 & 5621 & 15316 & 14596 & 16117 & 15397 & 15758 \\
\hline $\mathrm{SEm} \pm$ & - & - & 16.7 & 3.3 & 7.2 & 6.5 & 7.1 & 6.6 & 5.5 \\
\hline $\mathrm{CD}(\mathrm{P}=\mathbf{0 . 0 5})$ & - & - & 50 & NS & NS & NS & NS & NS & NS \\
\hline
\end{tabular}

*Statistically not analysed 
Table.4 Accumulated Photo thermal units $\left({ }^{0} \mathrm{C}\right.$ day hrs $)$ at different phenophases of chickpea as influenced by crop residue incorporation, time of sowing and irrigation

\begin{tabular}{|c|c|c|c|c|c|c|c|c|c|}
\hline \multirow[t]{2}{*}{ Treatment } & \multicolumn{2}{|c|}{ Emergence* } & \multicolumn{2}{|c|}{ Fifty \% flowering } & \multicolumn{2}{|c|}{$\begin{array}{l}\text { Physiological } \\
\text { maturity }\end{array}$} & \multicolumn{3}{|c|}{ Harvest } \\
\hline & $\begin{array}{l}\text { Rabi, } \\
2018\end{array}$ & $\begin{array}{l}\text { Rabi, } \\
2019\end{array}$ & $\begin{array}{l}\text { Rabi, } \\
2018\end{array}$ & $\begin{array}{l}\text { Rabi, } \\
2019\end{array}$ & $\begin{array}{r}\text { Rabi, } \\
2018\end{array}$ & $\begin{array}{l}\text { Rabi, } \\
2019\end{array}$ & $\begin{array}{l}\text { Rabi, } \\
2018\end{array}$ & $\begin{array}{l}\text { Rabi, } \\
2019\end{array}$ & Pooled \\
\hline \multicolumn{10}{|l|}{ Crop residue incorporation } \\
\hline $\mathrm{C}_{1}$ : Foxtail millet & 1101 & 989 & 9557 & 9359 & 19800 & 19749 & 20986 & 20978 & 20982 \\
\hline $\mathrm{C}_{2}$ : Greengram & 1101 & 988 & 9556 & 9356 & 19797 & 19747 & 20982 & 20977 & 20979 \\
\hline $\mathrm{C}_{3}:$ Fallow & 1099 & 986 & 9547 & 9355 & 19795 & 19741 & 20980 & 20974 & 20977 \\
\hline SEm \pm & - & - & 4.5 & 3.3 & 3.4 & 2.6 & 3.3 & 2.8 & 5.2 \\
\hline $\mathrm{CD}(\mathrm{P}=\mathbf{0 . 0 5})$ & - & - & NS & NS & NS & NS & NS & NS & NS \\
\hline \multicolumn{10}{|l|}{ Time of sowing } \\
\hline$D_{1}:$ October $2^{\text {nd }} F N$ & 1461 & 1161 & 10692 & 9880 & 22350 & 22169 & 23804 & 22169 & 22986 \\
\hline$D_{2}:$ November $1^{\text {st }} F N$ & 1182 & 1045 & 9709 & 9596 & 21923 & 21529 & 23611 & 22529 & 23070 \\
\hline$D_{3}$ : November $2^{\text {nd }} F N$ & 1041 & 1015 & 9638 & 9083 & 21769 & 21060 & 23211 & 21873 & 22541 \\
\hline $\mathrm{D}_{4:}$ December $1^{\text {st }}$ FN & 908 & 910 & 8462 & 8594 & 20475 & 19989 & 22256 & 21042 & 21649 \\
\hline SEm \pm & - & - & 5.7 & 6.2 & 12.5 & 6.7 & 12.1 & 11.5 & 12 \\
\hline $\mathrm{CD}(\mathrm{P}=0.05)$ & - & - & 17 & 18 & 38 & 20 & 37 & 35 & 37 \\
\hline \multicolumn{10}{|l|}{ Time of irrigation } \\
\hline$I_{1}$ : Irrigation at pre-flowering stage & - & - & 9712 & 9422 & 19866 & 19850 & 21786 & 21007 & 21396 \\
\hline $\begin{array}{l}\text { I }_{2} \text { : Irrigation at pod development } \\
\text { stage }\end{array}$ & - & - & 9711 & 9416 & 19859 & 19852 & 21782 & 21009 & 21396 \\
\hline $\begin{array}{l}\text { I }_{3} \text { : Irrigation at pre-flowering and pod } \\
\text { development stage }\end{array}$ & - & - & 9716 & 9418 & 19861 & 19849 & 21787 & 21007 & 21397 \\
\hline $\mathrm{SEm} \pm$ & - & - & 12.6 & 6.2 & 5.9 & 3.4 & 7.4 & 11.7 & 4.5 \\
\hline $\mathrm{CD}(\mathbf{P}=\mathbf{0 . 0 5})$ & - & - & NS & NS & NS & NS & NS & NS & NS \\
\hline
\end{tabular}


Table.5 Seed yield and harvest index of chickpea as influenced by crop residue incorporation, time of sowing and irrigation

\begin{tabular}{|c|c|c|c|c|c|c|}
\hline \multirow[t]{2}{*}{ Treatments } & \multicolumn{3}{|c|}{ Seed yield $\left(\mathrm{kg}^{-1}\right)$} & \multicolumn{3}{|c|}{ Harvest Index (\%) } \\
\hline & $\begin{array}{l}\text { rabi, } \\
2018\end{array}$ & $\begin{array}{l}\text { rabi, } \\
2019\end{array}$ & Pooled & $\begin{array}{l}\text { rabi, } \\
2018\end{array}$ & $\begin{array}{l}\text { rabi, } \\
2019\end{array}$ & Pooled \\
\hline \multicolumn{7}{|c|}{ Crop residue incorporation } \\
\hline $\mathrm{C}_{1}$ : Foxtail millet & 1229 & 1867 & 1546 & 46.72 & 44.31 & 45.53 \\
\hline $\mathrm{C}_{2}$ : Greengram & 1142 & 1828 & 1474 & 48.11 & 45.56 & 46.84 \\
\hline $\mathrm{C}_{3}$ : Fallow & 974 & 1447 & 1216 & 47.03 & 45.67 & 46.37 \\
\hline SEm \pm & 40.4 & 34.4 & 39 & 0.6 & 0.5 & 0.6 \\
\hline $\mathrm{CD}(\mathrm{P}=\mathbf{0 . 0 5})$ & 158 & 135 & 115 & NS & NS & NS \\
\hline \multicolumn{7}{|l|}{ Time of sowing } \\
\hline$D_{1}$ : October $2^{\text {nd }}$ FN & 1044 & 1700 & 1380 & 47.77 & 45.37 & 46.57 \\
\hline$D_{2}$ : November $1^{\text {st }} \mathbf{F N}$ & 1702 & 1957 & 1660 & 4470 & 43.50 & 44.21 \\
\hline$D_{3}$ : November $2^{\text {nd }} \mathbf{F N}$ & 1180 & 1770 & 1472 & 46.63 & 45.73 & 46.26 \\
\hline$D_{4:}$ December $1^{\text {st }}$ FN & 935 & 1429 & 1167 & 47.03 & 44.84 & 45.92 \\
\hline SEm \pm & 22.3 & 31.8 & 31 & 0.3 & 0.2 & 0.2 \\
\hline $\mathrm{CD}(\mathrm{P}=\mathbf{0 . 0 5})$ & 66 & 95 & 92 & 1.1 & 0.6 & 0.6 \\
\hline \multicolumn{7}{|l|}{ Time of Irrigation } \\
\hline $\begin{array}{l}I_{1} \text { : Irrigation at pre- } \\
\text { flowering stage }\end{array}$ & 801 & 1445 & 1117 & 47.55 & 45.04 & 46.31 \\
\hline $\begin{array}{l}\text { I }_{2} \text { : Irrigation at pod } \\
\text { development stage }\end{array}$ & 985 & 1633 & 1300 & 47.11 & 45.10 & 46.10 \\
\hline $\begin{array}{l}\text { I }: \text { Irrigation at pre- } \\
\text { flowering and pod } \\
\text { development stage }\end{array}$ & 1557 & 2064 & 1819 & 47.14 & 45.41 & 46.29 \\
\hline $\mathrm{SEm} \pm$ & 27.4 & 31.7 & 34 & 0.2 & 0.3 & 0.3 \\
\hline $\mathrm{CD}(\mathrm{P}=\mathbf{0 . 0 5})$ & 78 & 90 & 101 & NS & NS & NS \\
\hline \multicolumn{7}{|l|}{ Interaction } \\
\hline \multicolumn{7}{|l|}{ C x D } \\
\hline $\operatorname{SEm} \pm$ & 38.5 & 55.2 & 22.5 & 0.6 & 0.6 & 0.9 \\
\hline $\mathrm{CD}(\mathrm{P}=\mathbf{0 . 0 5})$ & 115 & 164 & 96 & NS & NS & NS \\
\hline \multicolumn{7}{|l|}{ CxI } \\
\hline SEm \pm & 47.4 & 55.0 & 38.2 & 0.9 & 0.5 & 0.6 \\
\hline $\mathrm{CD}(\mathrm{P}=\mathbf{0 . 0 5})$ & NS & NS & NS & NS & NS & NS \\
\hline \multicolumn{7}{|l|}{ D x I } \\
\hline SEm \pm & 54.8 & 63.5 & 52.5 & 0.3 & 1.0 & 0.9 \\
\hline $\mathrm{CD}(\mathrm{P}=\mathbf{0 . 0 5})$ & NS & NS & NS & NS & NS & NS \\
\hline \multicolumn{7}{|l|}{ CxD I I } \\
\hline SEm \pm & 94.9 & 110.0 & 75.4 & 0.9 & 0.6 & 0.5 \\
\hline $\mathrm{CD}(\mathrm{P}=\mathbf{0 . 0 5})$ & NS & NS & NS & NS & NS & NS \\
\hline
\end{tabular}


Fig.1 Thermal use efficiency (TUE) of chickpea at harvest as influenced by crop residue incorporation, time of sowing and irrigation
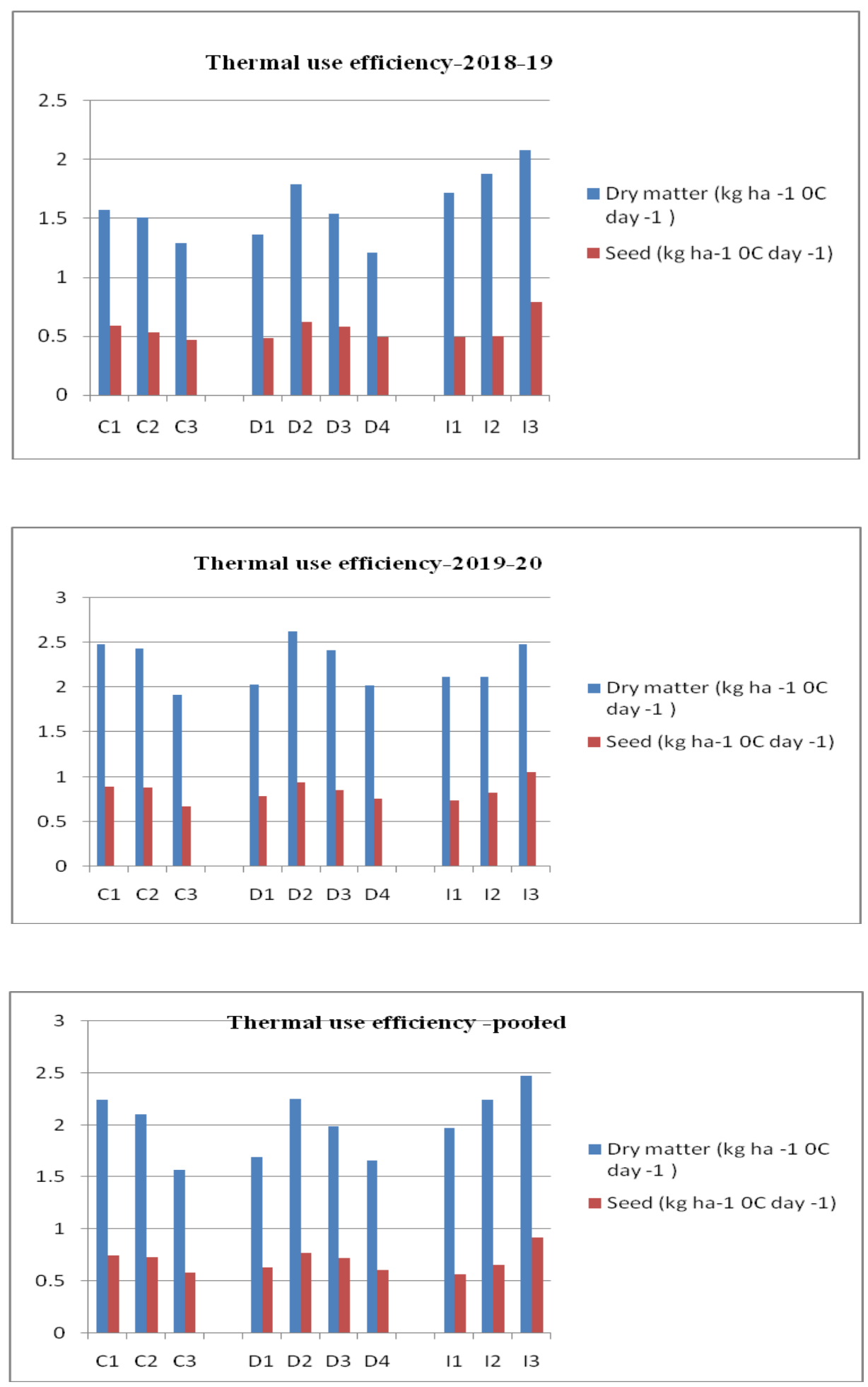


\section{Helio thermal units (HTU)}

Accumulated HTU valves were higher with October II fortnight sowing $\left(\mathrm{D}_{1}\right)$ and followed the same trend as of GDD from sowing to emergence, emergence to 50 per cent flowering and 50 per cent flowering to physiological maturity (Table 3). The HTU were influenced by time of sowing only but not with crop residues and irrigation, during both the years of study. The values of HTU were lower with late sown chickpea at each phenophase which indicates that crop facing suboptimal conditions which leads to lower yields. The interaction between crop residue incorporation, time of sowing and irrigation levels either at two level or three level interaction was not significant in altering the HTU of phenophases, during the both the years of study. These results are in close to the findings of Shamsi et al., (2011).

\section{Photo thermal units (PTU)}

The PTU values were influenced by time of sowing only but not with crop residues, irrigation or by their interaction, during both the years of study. Accumulated PTU valves were calculated and presented in Table 4 and results showed that the values were also higher with October $2^{\text {nd }}$ fortnight sowing $\left(D_{1}\right)$ and followed the same trend of GDD and HTU from sowing to emergence, emergence to 50 per cent flowering and 50 per cent flowering to physiological maturity. The values of PTU are lower with late sown chickpea at each phenophase which indicated that crop facing suboptimal conditions which leads to lower yields. The quantified PTU units were relatively lower during rabi 201920 due to cloudy weather and continuous rains.

\section{Seed yield}

Seed yield obtained were presented in Tabe. 5 and pooled data indicated that foxtail millet crop residue incorporation recorded higher chickpea seed yield (1546 kg ha ${ }^{-1}$ ) followed by greengram crop residue incorporation $\left(1474 \mathrm{~kg} \mathrm{ha}^{-1}\right)$. Fallow or no crop residue incorporation treatment recorded lowest seed yields $\left(1216 \mathrm{~kg} \mathrm{ha}^{-1}\right)$.

Pooled analysis of seed yield also indicated significant differences in time of sowing and irrigation as that observed in individual years. The pooled yield of $1660 \mathrm{~kg} \mathrm{ha}^{-1}$ was recorded with crop sown during November first fortnight $\left(D_{2}\right)$ of followed by that of November second fortnight $\left(D_{3}\right)$ sowing treatment. Seed yields followed increased trend up to November sowings and decreased beyond November month. The response to irrigation levels also followed similar trend as in case of individual years.

Chickpea GDD, and heat units are positively related and first sown crop $\left(D_{1}\right)$ recorded higher values compared to rest of times of sowing crop. But seed yield was less than $\mathrm{D}_{2}$ sown chickpea crop because of higher GDD for sowing to emergence and comparable number of GDD for emergence to 50 per cent flowering stage than $\mathrm{D}_{2}$. More over during second year an amount of $74.8 \mathrm{~mm}$ of rainfall received in eight rainy days from 44 to 46 meteorological weeks, which coincided with germination to vegetative stage of October second fortnight $\left(\mathrm{D}_{1}\right)$ sown crop. Whereas November first fortnight sown $\left(\mathrm{D}_{2}\right)$ crop utilised the favourable weather condition and recorded highest yields. These results are also in concurrence with the findings of Sunil Kumar and Martin Luther, (2018).

\section{Harvest index}

Harvest index of chickpea was presented in Table 5. An insight in to the data indicated that there was no significant difference due to crop residue incorporation and irrigation as well as their interactions but statistically significant values were achieved with 
different times of sowing. Highest harvest index was recorded with October $2^{\text {nd }} \mathrm{FN}$ sowing, during both the years of study, which was followed by November $2^{\text {nd }} F N$, December, $1^{\text {st }} \mathrm{FN}$ and November $1^{\text {st }} \mathrm{FN}$ in order of decent. The harvest index values were in contrast to seed yields. The November $1^{\text {st }}$ FN resulted in highest seed yield recorded lowest harvest index. This showed that, still there was a possibility for yield increase with best performed time of sowing, when partitioning ability was increased towards sink.

\section{Thermal use efficiency (TUE)}

Data on thermal use efficiency of different treatments was presented in Fig. 1 showed that crop residue incorporation, time of sowing and irrigations were significantly influenced the thermal use efficiency, in both years of study and on pooled basis, but all interactions were failed to differ significantly. Both dry matter and seed $\left(\mathrm{kg} \mathrm{ha}^{-1}\right.$ per degree day) producing efficiency values were significantly higher with November $1^{\text {st }} \mathrm{FN}$ sowing followed by November $2^{\text {nd }} F N$ sowing, first time sowing $\left(\mathrm{D}_{1}\right)$ and fourth time of sowing $\left(\mathrm{D}_{4}\right)$ in order of decent, during both years of investigation and on pooled basis.

Crop growing ecological condition was the prime factor to utilise the heat energy for growth, development and harvest of economic yields. Thermal use efficiency was maximum when chickpea sown in of November $1^{\text {st }} \mathrm{FN}$ with high degree relation with increase in yields, during both years of study and on pooled basis and found to be optimum time of sowing for chickpea in rabi season under double cropping system in vertisols of Andhra Pradesh. These results are in close to the findings of Shamsi et al., (2011). When the crop was raised during November first fortnight, growth is more advanced than average development of chickpea crop.
In conclusion, the assessment of weather indices and seed yield of chickpea showed that, maximum seed yield of chickpea can be achieved when chickpea was sown during November $1^{\text {st }}$ FN, with incorporation of foxtail millet crop residue with two irrigations, one at pre flowering and another at pod development stage under double cropping system in vertisols of Andhra Pradesh.

\section{References}

Agarwal, A., Dintwa, E and Joshi, P. 2016. Analysis of agro-residue burning and present scenario in key areas of northern plains in India. International Journal of Advanced Research. 4(3): 14991509.

Dixit, J.P., Pillai, P.V.A and Namdeo, K.N. 1993. Response of chickpea (Cicer arietinum L.) to planting date and irrigation schedule. Indian Journal of Agronomy. 38(1): 121-123.

Kiran, B.A and Chimmad, C.P. 2015. Effect of temperature regions on phonological parameters, yield and yield components of chickpea. Karnataka journal of Agricultural Sciences. 28(2): 168-171.

Lev Yadun, S., Gopher, A and Abbo, S. 2000. The Cradle of Agriculture. Science. 288: 1062-1063.

Mohammed. A., Tana, T., Piara Singh., Diriba, K and Adamu, M. 2017. Management options for rainfed chickpea (Cicer arietinum L.) in northeast Ethiopia under climate change condition. Climate Risk Management. 16: 222-233.

Nuttonson,M. Y.1955. Wheat climate relationships and use of phenology in ascertaining the thermal and photothermal requirements of wheat. Published by American Institute of Crop Ecology, Washington D.C. P. 388.

Rajput, R.P. 1980. Response of soybean crop to climate and soil environment $P h . D$ 
Thesis. Indian Agricultural Research Institute, Pusa, New Delhi.

Sada Kumar, Patel, S.R., Jhaar Singh, Ganeswari and Dwarika Prasad, 2018. Study about different agrometeorological indices under different growing environmental conditions of Raipur district of Chhattisgarh. International Journal of Chemical Studies. 6(4) 2310-2313.

Shamsi, K., Kobrace, S and Rasekhi, B.2011. The effects of different planting densities on seed yield and quantitative traits of rainfed chickpea (Cicer arietinum L.) varieties. African Journal of Agricultural Research. 6(3): 655-659.

Sandhu, S.S., Hodges, H.F. 1971. Effect of photo period, light intensity and temperature on vegetative growth, flowering and seed production in chickpea (Cicer arietimum L.). Indian Journal of Agronomy. 63: 913-914.
Rogertson, M. J. and Zeinali, E. 2006. Modeling Chickpea growth and development phenological development. Field Crop Research 99: 1-13

Sunil Kumar, M and Martin Luther. 2018. Effect of different dates of sowing and irrigation on growth and yield of chickpea cultivation and evaluation of CROPGRO Chickpea model. Ph.D. thesis, Acharya N G Ranga Agricultural University, Lam, Guntur.

Summerfield, R.J. Minchin, F.R., Roberts, E.H., Hadley, P. 1980. The effect of photoperiod and air temperature on growth and yield of chickpea (Cicer airetinum L.) Proceedings of International workshop of Chickpea Improvement, Hyderabad, 28 February 2 March, 1979, Pantancheru, ICRISAT. Pp. 121-149.

Soltani, A., Hammer, G.L., Torabi, B.,

\section{How to cite this article:}

Prabhakar, K., V. Sumathi, T. Giridhar Krishna, P. Sudhakar, S. Jaffar Basha and Karuna Sagar, G. 2020. Ascertaining Weather Indices to Exploit the Yield Potential of Chickpea (Cicer arietinum L.) at Scarce Rainfall Zone of Andhra Pradesh. Int.J.Curr.Microbiol.App.Sci. 9(11): 1534-1546. doi: https://doi.org/10.20546/ijcmas.2020.911.182 